values of $\Gamma_{A B}^{\infty}$ and $K_{A B}$ obtained from a plot similar to Fig. 3 are represented by a solid line in Fig. 2. The calculated result approximately agrees with the observed values.

The interfacial areas occupied by one molecule of C-24 in water-C-24 system and of $\mathrm{HgCl}_{2} \cdot \mathrm{C}-24$, which were calculated from the values of $\Gamma_{B}^{\infty}$ and $\Gamma_{A B}^{\infty}$, are 87 and $89 \mathrm{~A}^{2} /$ molecule, respectively. These values nearly correspond to the size of the crown ether ring of C-24 obtained from the molecular model. However, the value for $\mathrm{C}-24$ in $1 \mathrm{kmol} / \mathrm{m}^{3} \mathrm{NaCl}-\mathrm{C}-24$ system was calculated as $34 \mathrm{~A}^{2} /$ molecule, and the adsorbed state of $\mathrm{C}-24$ in this case is not clear.

\begin{tabular}{|c|c|}
\hline \multicolumn{2}{|c|}{ Nomenclature } \\
\hline$C_{i}$ & $\begin{array}{l}=\text { concentration of species } i \text { in organic phase } \\
\qquad\left[\mathrm{mol} / \mathrm{m}^{3}\right]\end{array}$ \\
\hline$K_{i}$ & $\begin{array}{l}=\text { adsorption equilibrium constant of species } i \\
\qquad\left[\mathrm{~m}^{3} / \mathrm{mol}\right]\end{array}$ \\
\hline$R$ & $=$ gas constant \\
\hline
\end{tabular}

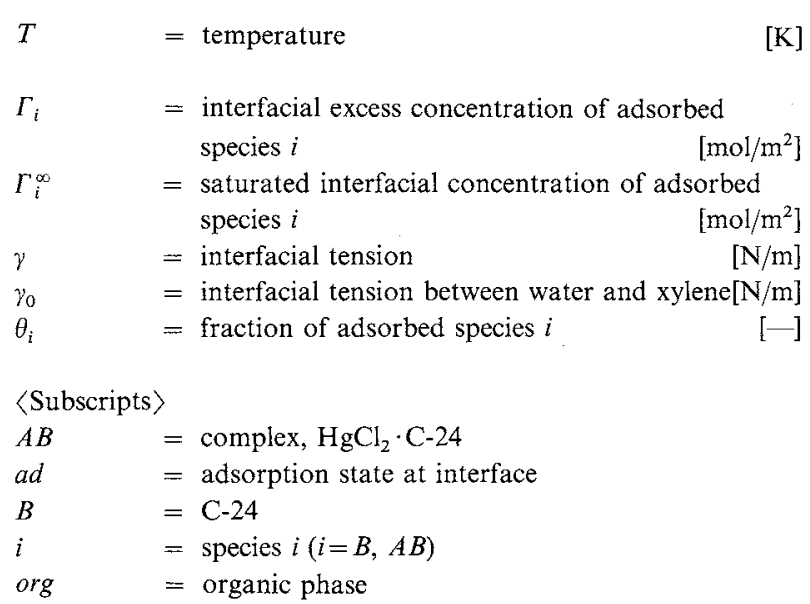

\section{Literature Cited}

1) Izatt, R. M., D. W. McBride, Jr., J. J. Christensen, J. S. Bradshaw and G. A. Clark: J. Membr. Sci., 22, 31 (1985).

2) Kataoka, T., T. Nishiki, T. Sasaki and D. Kato: J. Chem. Eng. Japan, 16, 459 (1983).

\title{
THEORETICAL ANALYSIS OF A NEW SEPARATION PROCESS UTILIZING EXTRACTION AND ENZYME REACTIONS
}

\author{
HARUO ISHIKAWA, HIROKAZU NISHIDA AND HARUO HIKITA \\ Department of Chemical Engineering, University of Osaka Prefecture, Sakai 591
}

\begin{abstract}
Key Words: Separation by Extraction, Enzyme Reaction, Immobilized Enzyme Membrane, Separation of Isomers, Boundary Value Problem, Finite Element Method
\end{abstract}

\section{Introduction}

Extraction is one of the important unit operations which utilize effectively the partitioning of components between two immiscible solvents or the difference of the partition coefficients between components. Therefore, a number of investigations on extraction have been carried out, and extensive efforts still continue for developing effective extraction processes and extractors. Recently some remarkable techniques have been introduced in this field. One of them is a liquid membrane technique first developed by $\mathrm{Li}^{3}{ }^{3)} \mathrm{A}$ number of liquid membrane processes have been demonstrated over the past ten years, and some of them are said to be near commercialization.

Received October 3, 1985. Correspondence concerning this article should be ad dressed to H. Ishikawa. H. Nishida is now with Osaka Prefectural Technical College, Neyagawa 572.
Introduction of enzymatic reactions has also brought promising effects. Removal of phenol in waste water ${ }^{4)}$ and separation of optical isomers ${ }^{5,7)}$ are typical separation processes in which enzyme reactions have been successfully combined with extraction or liquid membrane technique. However, the enzymes in most of these processes easily become deactivated, because they are simply confined in the aqueous phase.

The purpose of this work is to develop a new and effective extraction process to achieve both the separation and enrichment of valuable substances. The process consists of simultaneous conversion of objective components by enzymatic reaction and extraction of resulting products by solvents. For an idealized extractor, the performance has been analyzed numerically by using the matrix method. 


\section{Theory}

\subsection{Model formulation}

The extractor used in this work has an enzymebound porous catalytic membrane, which is in the aqueous phase adjacent to the interface between the aqueous and solvent phases. It is shown schematically in Fig. 1. It differs a little from the membrane reactors developed by Matson ${ }^{5}$ in which two fluid flows are separated by a composite of a permselective membrane and a catalytic membrane. An objective component $A$ which is ionized completely in the feed stream diffuses into the catalytic membrane where an enzyme is immobilized to convert $A$ into an unionized product $B$ as:

$$
A \stackrel{\text { enzyme }}{\longrightarrow} B
$$

and the rate of reaction is given by the following Michaelis-Menten equation:

$$
-r_{A}=\frac{V_{\max } C_{A}}{C_{A}+K_{m}}
$$

The rate equations for enzyme reactions are generally more complicated, but we consider that Eq. (2) is sufficient for our purpose of showing the usefulness of the process.

Almost all the reaction product $B$ is recovered in the sweep flow, as will be discussed later, if a solvent in which the product is easily soluble is chosen as the fluid for the sweep flow and if the extractor length is sufficiently long.

The basic differential equations for $A$ and $B$ in the catalytic membrane are given in dimensionless form as

$$
\begin{aligned}
& \frac{d^{2} A}{d \xi^{2}}=\phi_{1}^{2} \frac{K_{M} A}{A+K_{M}} \\
& \frac{d^{2} B}{d \xi^{2}}=-\psi \phi_{1}^{2} \frac{K_{M} A}{A+K_{M}}
\end{aligned}
$$

and the boundary conditions are given by

$$
\begin{array}{ll}
\xi=0 ; & A=A_{F}, \quad B=B_{F} \\
\xi=1 ; & A=A_{P} / K_{p A}, \quad B=B_{P} / K_{p B}
\end{array}
$$

For simplicity, we assume that both the feed and the sweep flows are plug flows and the mass transfer resistance in both the flows is negligible. Then the basic mass balance equations for $A$ and $B$ in the flows are expressed as

$$
\begin{array}{ll}
\frac{d A_{F}}{d \Phi}=-\tilde{N}_{A F}, & \frac{d B_{F}}{d \Phi}=-\tilde{N}_{B F} \\
\frac{d A_{P}}{d \Phi}=-\sigma \theta \tilde{N}_{A P}, & \frac{d B_{P}}{d \Phi}=-\sigma \theta \tilde{N}_{B P}
\end{array}
$$

where $\sigma$ indicates an integer and is equal to plus one

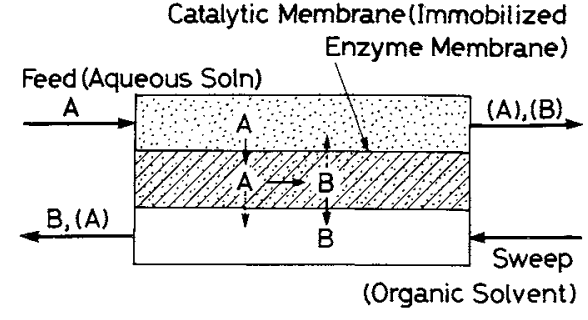

Fig. 1. Counter-current extractor fitted with immobilized enzyme membrane.

$(+1)$ for a counter-current flow and minus one $(-1)$ for a co-current flow. $\tilde{N}_{A F}, \tilde{N}_{B F}, \cdots$ in Eqs. (7) (10) are the dimensionless mass fluxes at the boundaries and are defined by

$$
\begin{array}{ll}
\tilde{N}_{A F}=-\left.\frac{d A}{d \xi}\right|_{\xi=0}, & \tilde{N}_{B F}=-\left.\frac{1}{\psi} \cdot \frac{d B}{d \xi}\right|_{\xi=0} \\
\tilde{N}_{A P}=-\left.\frac{d A}{d \xi}\right|_{\xi=1}, & \tilde{N}_{B P}=-\left.\frac{1}{\psi} \cdot \frac{d B}{d \xi}\right|_{\xi=1}
\end{array}
$$

The boundary conditions for Eqs. (7) (10) are given by

$$
\begin{aligned}
& \Phi=0 ; \quad A_{F}=1, \quad B_{F}=0 \\
& \Phi=\frac{(1+\sigma)}{2} \Phi_{T} ; \quad A_{P}=0, \quad B_{P}=0
\end{aligned}
$$

\subsection{Numerical method}

Equations (3), (4) and (7)-(14) must be solved numerically with boundary conditions given by Eqs. (5), (6), (15) and (16), because they cannot be solved analytically. As can be seen from the boundary conditions, the problem for a counter-current extractor becomes the boundary value problem for both the directions of diffusion and fluid flow. The problem of this type can be solved by the matrix method, and both the finite difference and the finite element approximations can be applied to transform the above equations into matrix forms. In this work, however, the latter approximation is used because of its ease of handling boundary conditions and its broad applicability for various geometries.

A computer algorithm for solving the matrix equations has been developed (see Appendix).

\section{Results and Discussion}

In the special case in which the reaction is irreversible first order $\left(K_{m} / C_{A F O} \gg 1\right)$, the concentration profiles of the reactant $A$ and the product $B$ in both the flows can be obtained analytically. The results of the numerical analysis for this special case were compared with the analytical solutions, and were found to agree well with them within an error of less than $1 \%$ when the numbers of elements were chosen as $m=10$ and $n=40$. From this result, the numerical method de- 


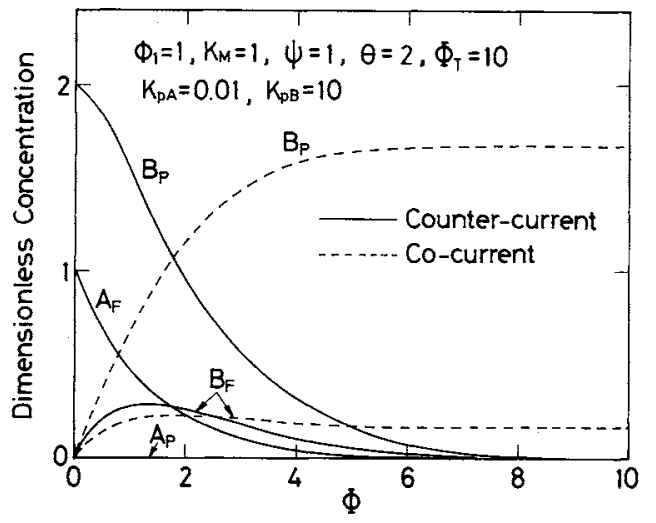

Fig. 2. Concentration profiles of reactant $A$ and product $B$ in feed and sweep flows.

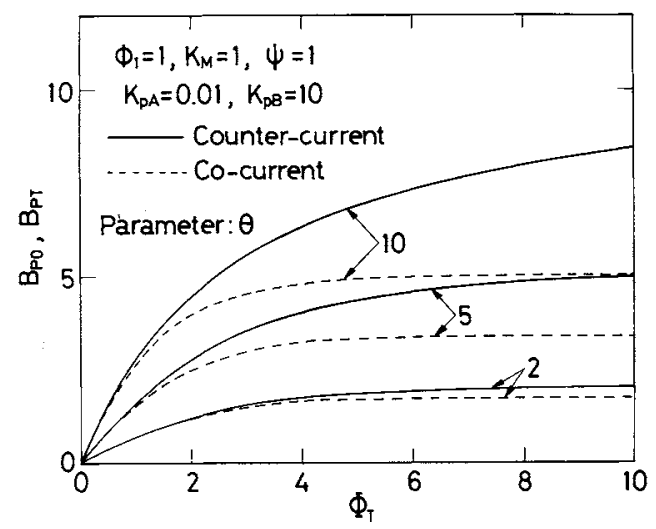

Fig. 3. Effect of dimensionless space time $\Phi_{T}$ and liquid flow rate ratio $\theta$ on concentration of $B$ in sweep flow at extractor exit.

veloped in this work is considered to have sufficient accuracy to solve the boundary-value problems.

Figure 2 shows typical concentration profiles of $A$ and $B$ in the feed and the sweep flows. As can be seen in the figure, almost all the product $B$ is recovered in the sweep flow, and its concentration at the exit of the extractor is higher for a counter-current flow than for a co-current flow.

Figure 3 shows the effect of the dimensionless space time $\Phi_{T}$ and the liquid flow rate ratio $\theta$ on the dimensionless concentrations $B_{P T}$ and $B_{P 0}$ of $B$ at the exit in the sweep flow. The figure shows that both $B_{P T}$ and $B_{P 0}$ increase with an increase in the parameter $\Phi_{T}$, which is directly proportional to the total surface area $S_{T}$ of the catalytic membrane and is inversely proportional to the flow rate $V_{F}$ of the feed solution, and that they approach asymptotically the limiting value given by

$$
B_{P 0}=\theta \quad \text { (counter-current extractor) }
$$

or

$$
B_{P T}=\frac{\theta}{1+\theta / K_{p B}} \quad \text { (co-current extractor) }
$$

From the figure and Eqs. (17) and (18), it was found that the concentration of $B$ recovered in the sweep flow increases with $\theta$. Furthermore, when the value of $\Phi_{T}$ is large and the value of $K_{p B}$ is not very large, performance of the counter-current extractor was found to be much superior to that of the co-current one from the standpoint of product concentration. The results shown above indicate that component $A$ in the feed solution can be recovered in the sweep solution as a form of $B$ in a high concentration.

The above process can be modified by replacing the aqueous solution as the fluid of the feed flow by an organic solvent and the organic solvent as a fluid of the sweep flow by an aqueous solution. In this case, the unionized components in the feed flow consisting of the organic solvent dissolve in the aqueous phase of the porous catalytic membrane and hydrolyze into ionized products by enzyme reaction. The ionized products cannot be reextracted by the organic solvent and are recovered in the sweep flow of the aqueous solution.

These processes may be suitable for separation of various isomers such as a mixture of derivatives of $\alpha$ and $\beta$-naphthols ${ }^{6)}$ (positional isomers) and a mixture of $\mathrm{D}$ - and L-amino acid derivatives ${ }^{1)}$ (optical isomers). In the former example, $\beta$-naphthylsulfate (component $A$ ) in the feed solution containing both $\alpha$ and $\beta$-naphthylsulfates will selectively be hydrolyzed into $\beta$-naphthol (component $B$ ) by enzyme sulfatase, then extracted by the organic solvent and separated from the unreacted $\alpha$-naphthylsulfate in the aqueous phase. When sulfatase from Helix pomatia is used as a catalyst, the rate of hydrolysis of $\beta$-naphthylsulfate may be approximately expressed by Eq. (2) according to Pelsy and Klibanov. ${ }^{6}$ Therefore, we will be able to do separation experiments by using decanol as a fluid of the sweep flow under conditions of parameters similar to those shown in Fig. 3, and to obtain similar results for the separation and the recovery of $\beta$ naphthol.

In the present process, the selection of the organic solvent as a fluid of the feed or the sweep flow is important. First of all, we must choose such solvents that do not deactivate the enzyme. Then, to ensure a high enrichment of the product in the process shown in Fig. 1, we should select a solvent that has a large partition coefficient for the product and a small partition coefficient for the reactant. Decanol may be a suitable solvent because decanol dissolved in water activates some enzymes such as $\alpha$-chymotripsin ${ }^{5)}$ and fumarase. $^{2)}$

As mentioned above, the extraction process developed in this work may be used effectively to separate objective components from a mixture and enrich them simultaneously. 


\section{Appendix}

In this work, the catalytic membrane and the flow paths were divided into a number of segment elements for the diffusional and the flow directions, respectively. The finite elements and the nodes in the membrane were numbered from the interface between the feed stream and the membrane, and those in the flow paths were from the inlet of the feed stream.

First, we obtained the finite element equations for component $A$ in the membrane by applying the Galerkin method ${ }^{8)}$ to the basic differential equation Eq. (3). For the $i$-th element at $\Phi_{j}$, the equations are given by

$$
\left.\begin{array}{l}
-\frac{A_{i+1, j}-A_{i, j}}{h}+\frac{h}{2} \phi_{1}^{2} \frac{K_{M} A_{i, j}}{K_{M}+A_{i, j}}=-\left.\frac{d A}{d \xi}\right|_{\xi_{i}, \Phi_{j}} \\
\frac{A_{i+1, j}-A_{i, j}}{h}+\frac{h}{2} \phi_{1}^{2} \frac{K_{M} A_{i+1, j}}{K_{M}+A_{i+1, j}}=\left.\frac{d A}{d \xi}\right|_{\xi_{i+1}, \Phi_{j}}
\end{array}\right\} \quad(i=1, \cdots, m)
$$

where $\xi_{i}$ and $\Phi_{i}$ are given by

$$
\xi_{i}=(i-1) h, \quad \Phi_{j}=(j-1) l
$$

The second terms of the left-hand side in Eq. $(A-1)$ were derived by integration using the trapezoidal rule. The terms of the right-hand side of Eq. (A-1) disappear except for two terms, $-\left.(d A / d \xi)\right|_{\xi_{1}, \Phi_{j}}$ and $\left.(d A / d \xi)\right|_{\xi_{m+1}, \Phi_{i}}$, when the finite element equations for all elements for $i=1, \cdots, m$ are combined into a matrix equation.

Next, we obtained the finite element equations for $A$ in the feed flow by applying the Galerkin method to Eqs. (7) and (11). For the $j$-th element, the equations are given by

$$
\left.\begin{array}{l}
\frac{A_{F j+1}-A_{F j}}{l}=\left.\frac{d A}{d \xi}\right|_{\xi_{1}, \Phi_{j}} \\
\frac{A_{F j+1}-A_{F j}}{l}=\left.\frac{d A}{d \xi}\right|_{\xi_{1}, \Phi_{j+1}}
\end{array}\right\}(j=1, \cdots, n)
$$

Similarly, Eqs. (9) and (13) can be converted to

$$
\left.\begin{array}{c}
\frac{1}{\sigma \theta} \cdot \frac{A_{P j+1}-A_{P_{j}}}{l}=\left.\frac{d A}{d \xi}\right|_{\xi_{m+1}, \Phi_{j}} \\
\frac{1}{\sigma \theta} \cdot \frac{A_{P j+1}-A_{P j}}{l}=\left.\frac{d A}{d \xi}\right|_{\xi_{m+1}, \Phi_{j+1}}
\end{array}\right\}(j=1, \cdots, n)
$$

All the element equations for all the elements for $j=1, \cdots, n$ can be combined into a matrix form by the same procedure mentioned above.

Finally, all the matrix equations derived above are combined by taking into consideration the following relations:

$$
A_{F j}=A_{1, j}, \quad A_{P j}=K_{p A} A_{m+1, j}
$$

and the boundary conditions

$$
\left.\begin{array}{l}
A_{1,1}=1 \\
A_{m+1, n+1}=0 \quad(\sigma=+1) \\
A_{m+1,1}=0 \quad(\sigma=-1)
\end{array}\right\}
$$

The resulting total governing matrix equation for component $A$ can be expressed as

$$
\left[S_{A}\right]\{A\}+\{F\}=\{0\}
$$

Similarly, the total matrix equation for component $B$ is given by

$$
\left[S_{B}\right]\{B\}-\psi\{F\}=\{0\}
$$

where $\left[S_{B}\right]$ can be obtained by replacing $K_{p A} / \sigma \theta$ by $\psi K_{p B} / \sigma \theta$ in $\left[S_{A}\right]$ of Eq. (A-10) and the boundary conditions are given by

$$
\left.\begin{array}{c}
B_{1,1}=0 \\
B_{m+1, n+1}=0 \quad(\sigma=+1) \\
B_{m+1,1}=0 \quad(\sigma=-1)
\end{array}\right\}
$$

Equations (A-10) and (A-11) are nonlinear simultaneous equations and were solved by the Newton method. The procedure for solving the equations is as follows.

(1) Let $k$ be $k=1$ and assume $\{A\}_{k}$

(2) Obtain the incremental vector $\{\Delta A\}_{k}$ by solving the following equation:

$$
\left(\left[S_{A}\right]+[J F]_{k}\right)\{\Delta A\}_{k}=-\left[S_{A}\right]\{A\}_{k}-\{F\}_{k}
$$

where $[J F]_{k}$ expresses the Jacobi matrix of $\{F\}_{k}$.

(3) Calculate $\{A\}_{k+1}$, which is the $(k+1)$-th approximation of $\{A\}$, from

$$
\{A\}_{k+1}=\{A\}_{k}+\{\Delta A\}_{k}
$$

(4) Proceed to the next step with $\{A\}=\{A\}_{k+1}$, if $\left|\{A A\}_{k}\right|<\varepsilon$, and go back to step (2) with $k=k+1$, if not.

(5) Calculate $\{B\}$ by solving Eq. (A-11).

\section{Nomenclature}

dimensionless concentration of reactant $A$, $C_{A} / C_{A F 0}$

$=$ dimensionless concentration of product $B$, $C_{B} / C_{A F O}$

$D_{e} \quad=$ effective diffusivity $\quad\left[\mathrm{m}^{2} \cdot \mathrm{s}^{-1}\right]$ $\left[\mathrm{mol} \cdot \mathrm{m}^{-3}\right]$

$F \quad=$ column vector consisting of reaction term $[-]$

$h \quad=$ size of element, $1 / m$

$K_{M} \quad=$ dimensionless Michaelis constant, $K_{m} / C_{A F 0} \quad[-]$

$K_{m} \quad=$ Michaelis constant $\quad\left[\mathrm{mol} \cdot \mathrm{m}^{-3}\right]$

$K_{p} \quad=$ partition coefficient $\quad[-]$

$l \quad=$ size of element, $\Phi_{T} / n \quad[-]$

$m \quad=$ number of element for diffusional direction $\quad[-]$

$N \quad=$ mass flux $\quad\left[\mathrm{mol} \cdot \mathrm{m}^{-2} \cdot \mathrm{s}^{-1}\right]$

$\tilde{N} \quad=$ dimensionless mass flux $\delta N / C_{A F 0} D_{e A} \quad[-]$

$n \quad=$ number of element for flow direction $\quad[-]$

$S \quad=$ surface area of catalytic membrane measured from extractor inlet

$\left[\mathrm{m}^{2}\right]$

$S_{A} \quad=$ element coefficient matrix for $A$

$S_{B} \quad=$ element coefficient matrix for $B$

$V \quad=$ liquid flow rate $\quad\left[\mathrm{m}^{3} \cdot \mathrm{s}^{-1}\right]$

$V_{\max }=$ maximum reaction rate of enzyme reaction $\left[\mathrm{mol} \cdot \mathrm{m}^{-3} \cdot \mathrm{s}^{-1}\right]$

$x=$ distance from surface of catalytic membrane $[\mathrm{m}]$

$=$ thickness of catalytic membrane

$[\mathrm{m}]$

$=$ ratio of liquid flow rates, $V_{F} / V_{P}$

$=$ dimensionless distance, $x / \delta$

$=$ integer number, +1 or -1

$[-]$

$=$ dimensionless space time, $D_{e A} S / \delta V_{F}$

$[-]$

$\phi_{1} \quad=$ Thiele modulus, $\delta \sqrt{V_{\max } / K_{m} D_{e A}}$

$[-]$

$\psi \quad=$ diffusivity ratio, $D_{e A} / D_{e B}$

$[-]$

〈Subscripts〉

$\begin{array}{ll}A & =\text { reactant } \\ B & =\text { product } \\ F & =\text { feed stream } \\ i, j & =\text { nodal point } \\ k & =\text { number of iteration } \\ P & =\text { sweep or product stream } \\ T & =\text { outlet of extractor } \\ 0 & =\text { inlet of extractor }\end{array}$




\section{Literature Cited}

1) Bergman, M. and H. Frankel-Conrat: J. Biol. Chem., 119, 707 (1937).

2) Ishikawa, H., T. Tanaka, K. Kurose and H. Hikita: to be published.

3) Li, N. N.: AIChE J., 17, 459 (1971).

4) May, S. W. and N. N. Li: "Enzyme Engineering," E. K. Pye and L. B. Wingard, Eds., Vol. 2, Plenum Press, New York (1974):
5) Matson, S. L.: Ph. D. Dissertation, Univ. of Pennsylvania, Philadelphia, U.S.A. (1979).

6) Pelsy, G. and A. M. Klibanov: Biotech. Bioeng., 25, 919 (1983).

7) Scheper, Th., W. Halwachs and K. Schügerl: Chem. Eng. J., 29, B31 (1984).

8) Tsukada, T., M. Hozawa, N. Imaishi and K. Fujinawa: J. Chem. Eng. Japan, 17, 246 (1984).

\title{
INFLUENCE OF DONOR SOLUTION UPON SKIN PERMEATION OF DRUG
}

\author{
K. TOJO, C. C. CHIANG AND 'Y. W. CHIEN \\ Controlled Drug Delivery Research Center, Rutgers University, College of Pharmacy, \\ Piscataway, New Jersey 08854, U.S.A.
}

Key Words: Transdermal Drug Delivery, Percutaneous Absorption, Progesterone, Polyethylene Glycol, Azone

\section{Introduction}

It is well known that the intrinsic permeation rate of drug through a polymeric membrane is usually independent of the donor and receptor solutions used in the in vitro diffusion cell, because the drug solubility and the drug diffusivity in the membrane are hardly affected by the donor or receptor solutions used. The difference in apparent rate of permeation caused by different donor and receptor solutions used could be attributed to the difference in the diffusion boundary layer, which is influenced by the mixing conditions of the solution, and the partitioning of drug on the surface of the membrane. ${ }^{5)}$

Theeuwes et al. also discussed the effect of solution used in an in vitro skin permeation study of the rate of drug permeation and concluded that the steady-state rate of drug permeation is independent of the elution medium in in vitro permeation experiments. ${ }^{3)}$ This conclusion should carefully be examined, since a biological membrane such as the skin is usually very sensitive to environmental conditions.

In this communication, we demonstrate the effect of the donor solution on the rate of skin permeation of progesterone, using a hydrophilic or hydrophobic solution.

\section{Experimental}

The in vitro skin permeation system used in this

Received October 18, 1985. Correspondence concerning this article should be addressed to K. Tojo. study is the same as described previously. ${ }^{1)}$ The hydrodynamic characteristics of this diffusion cell has already been established by the present authors. ${ }^{4)}$

Water, aqueous polyethylene glycol 400 solution $(10-40 \%)$ and silicone fluid (DC 360, 0.02 Pa.s) were used as the donor solution, while the receptor solution was always $40 \%$ PEG 400 solution. An excess amount of progesterone crystals was suspended in the donor solution to maintain a constant saturated concentration during the entire period of the permeation experiment.

At predetermined time intervals, 40-microliter samples were withdrawn from the receptor compartment and assayed by a HPLC procedure.

The saturated concentration of progesterone in various donor solutions was measured by an $\mathrm{UV} /$ vis spectrophotometer for the aqueous PEG 400 solutions and by an extraction method using methanol ${ }^{2)}$ for the silicone fluid. All the skin permeation experiments were carried out at $37^{\circ} \mathrm{C}$.

Abdominal hairless mouse skin (the intact skin or the stripped skin without stratum corneum, female, 5-7 weeks) excised was quickly mounted between the diffusion half-cells and the concentration of progesterone in the receptor solution was monitored. The stripped skin was prepared by 16-time stripping using a cellophane tape. ${ }^{6,7)}$

The effect of the diffusion boundary layer on the rate of skin permeation was corrected by a method recently proposed ${ }^{5)}$ and the intrinsic permeation rate was evaluated. 